\title{
Surgical Stabilization of Femur Fractures in Post-Traumatic Hypoxemic Patients: When and Why?
}

\author{
Ramesh Kumar Sen ${ }^{1}$; Goverdhan Dutt Puri ${ }^{2}$; Indu Mohini ${ }^{2, *}$; Anil Pratap ${ }^{1}$; Nirmal Raj ${ }^{1}$ \\ ${ }^{1}$ Department of Orthopedic Surgery, Post Graduate Institute of Medical Education and Research, Chandigarh, India \\ ${ }^{2}$ Department of Anaesthesia and Intensive Care, Post Graduate Institute of Medical Education and Research, Chandigarh, India \\ ${ }^{*}$ Corresponding author: Indu Mohini, Department of Anesthesia and Intensive Care, Post Graduate Institute of Medical Education and Research, 160012, Chandigarh, India. Tel: +91- \\ 1722686677, E-mail: indumohini@gmail.com
}

Received: October 15, 2013; Revised: May 27, 2014; Accepted: June 26, 2014

\begin{abstract}
Background: Post-traumatic hypoxemia can deteriorate during operative manipulations.
Objectives: In the present study, criteria-based approach was applied to determine optimum conditions for femur surgery. The aim of this study was to optimize perioperative management of post-traumatic hypoxemia.

Patients and Methods: In this prospective observational study, post-traumatic adults with $\mathrm{PaO}_{2}<70 \mathrm{mmHg}$ in room air were enrolled. Physiological parameters, $\mathrm{O}_{2}$ saturation ( $\mathrm{SO}_{2}$ ), arterial blood gas (ABG) analysis, Schonfeld fat embolism index score (SS), and Murray's lung injury scores (LIS) were assessed. The management protocol was femur surgery when patient was hemodynamically stable with LIS $<$ 2.5 and $\mathrm{PaO}_{2} / \mathrm{FiO}_{2}>200 \mathrm{mmHg}\left(\mathrm{FiO}_{2}<0.5, \mathrm{PEEP}<8 \mathrm{~cm} \mathrm{H}_{2} \mathrm{O}\right)$.

Results: A total of 31 adults ( 26 males and 5 females) with LIS of 0.1 to 2.5 (26 patients) and $>2.5$ (five patients) at admission were recruited. Sixteen patients were admitted within 24 hours and 15 between 24 and 90 hours after injury. Thirteen patients were operated within 24 hours. Post-operative LIS was improved. No adverse sequels or mortality were seen.

Conclusions: Appropriate surgical stabilization can be safely performed during established post-traumatic hypoxemia using a multidisciplinary approach, continuous monitoring, and serial investigations to diagnose fulminant pathology and associated injuries.
\end{abstract}

Keywords: Post-traumatic hypoxemia; Damage-control surgery

\section{Background}

Post-traumatic hypoxemia and fat embolism syndrome (FES) can lead to a cascade of events resulting in acute lung injury $(1,2)$. Although operative stabilization is thought to control the progress of disease (3-6), release of marrow fat at the time of surgical manipulation can deteriorate the pulmonary status of these patients in whom neutrophil activation and adhesions are already causing damage to the alveoli, thus, making a vicious cycle (secondary insult) (7-9). Various surgical techniques have been developed to reduce the extravasation of fat and to block the inflammatory cascade of events (10); however, optimum time of surgery in patients with established hypoxemia has not been determined yet. In this study, all the participants underwent operative stabilization after meeting predefined hemodynamic and respiratory criteria. They were subsequently evaluated for the clinical outcome and adverse events.

\section{Objectives}

Post-traumatic hypoxemia can deteriorate during operative manipulations. In the present study, criteria-based approach was applied to determine optimum conditions for femur surgery. The aim of this study was to optimize perioperative management of patients with post-traumatic hypoxemia.

\section{Patients and Methods}

After achieving an approval of the Institute Ethical Committee and obtaining written informed consent from participants, this prospective observational study was performed on patients aged 18 to 45 years with new injury severity score (NISS) (11) of more than nine and significant post-traumatic hypoxemia who were admitted for surgical stabilization of femur fractures. Patients with associated cerebral or thoracoabdominal injuries, systemic illnesses, or chronic smokers were excluded. Patients were labeled as hypoxemic if their $\mathrm{PaO} 2$ was less than 70 $\mathrm{mmHg}$ in room air and as clinical FES if Schonfeld's fat embolism index score (SS) (12) was more than five. Time intervals between the injury and arrival to the hospital and duration of hypoxemia were recorded. All these patients had an intensive care therapy back-up and received supplemental oxygen using appropriate ventilatory strategy. Electrocardiography, noninvasive and invasive blood pressure monitoring, arterial oxygen saturation $\left(\mathrm{SO}_{2}\right)$, end-tidal carbon dioxide $\left(\mathrm{EtCO}_{2}\right)$, central venous pressure (CVP), temperature, and hourly urine output measurement were used to monitor the patient. Intra-

Copyright (c) 2014, Kashan University of Medical Sciences; Published by Kowsar Corp. This is an open-access article distributed under the terms of the Creative Commons Attribution License, which permits unrestricted use, distribution, and reproduction in any medium, provided the original work is properly cited. 
venous fluids and/or whole blood were administered to maintain the CVP at $12 \pm 2 \mathrm{mmHg}$. Detailed history regarding physical health, coexisting medical problems, current medications, allergies, and previous anesthetic or surgical experience were recorded. Glasgow Coma Score (GCS) and vital signs were determined during this period at regular intervals. Performed standard investigations included arterial blood gas (ABG) analysis, complete hemogram, serum electrolytes, liver and renal function tests, serum lactate, chest roentgenogram and 12-lead ECG. Modified Murray's Lung injury scoring (LIS) (12) was done repeatedly every four hours to quantify the respiratory dysfunction (Appendix 1) (13). Patients were scheduled for operative stabilization as soon as they achieved hemodynamic stability (without vasopressors and/or inotropic support), normothermic status, $\mathrm{PaO}_{2} / \mathrm{FiO}_{2}$ ratio of more than $200 \mathrm{mmHg}$ at $\mathrm{FiO}_{2}$ of less than 0.5 , positive end-expiratory pressure (PEEP) of less than $8 \mathrm{~cm} \mathrm{H}_{2} \mathrm{O}$, and LIS score of more than 2.5. It was hypothesized that carefully performed operative stabilization would not affect clinical severity of hypoxemia, would protect the patient from risk of recurrent embolization, and would improve overall recovery.

General anesthesia with endotracheal intubation was performed to maintain the $\mathrm{EtCO}_{2}$ between 30 to $35 \mathrm{mmHg}$. $\mathrm{ABG}$ analysis was done 30 minutes after surgical incision and regularly towards the end of surgery. The surgical stabilization of fractures was done by standard techniques of unreamed intramedullary nailing with distal venting and plating according to the fracture patterns. Surgery time was restricted to the minimum possible time for appropriate stabilization. Patients who were hemodynamically stable with $\mathrm{PaO} 2 / \mathrm{FiO} 2$ ratio of more than $300 \mathrm{mmHg}$ were reversed and extubated. Patients who were preoperatively on mechanical ventilation or hemodynamically unstable were electively ventilated. In the post-operative period, GCS, vital parameters, CVP, and $\mathrm{SO}_{2}$ were recorded at regular intervals. $\mathrm{ABG}$ and $\mathrm{SO}_{2}$ were assessed every twelve hours until the patients were weaned off the oxygen therapy (maintaining $\mathrm{PaO}_{2}>80 \mathrm{mmHg}$ on $\mathrm{FiO}_{2}<0.28$ ). Chest roentgenogram and LIS were assessed daily; persistent rise in LIS value by more than 0.5 or reduction in $\mathrm{PaO} 2 / \mathrm{FiO} 2$ ratio to less than $200 \mathrm{mmHg}$ were considered as deterioration and persistent fall in LIS by 0.5 was considered as improvement after surgery. Number of patients requiring mechanical ventilatory support, type of ventilatory strategy used, need for high PEEP $\left(>8 \mathrm{~cm} \mathrm{H}_{2} \mathrm{O}\right)$, and duration of mechanical ventilation were recorded. Total hospital stay, length of ICU stay, and morbidity and mortality during hospitalization were also determined. Perioperative adverse events like frank pulmonary edema, hypotension or hypertension requiring inotropes or vasoactive substances, respectively, excessive blood loss, and neurological or respiratory deterioration, ie, rise in LIS score by more than one, were recorded.

The data were analyzed with SPSS (v.10.1, SPSS Inc., Chicago, IL, USA). Patient characteristics were analyzed by the Chi square test for nominal data. Parametric data was analyzed using the independent-samples t-test. To evaluate anesthetic and surgical data, the independent-samples t-test or the Mann Whitney U-test were used. The occurrence of postoperative events were analyzed with the Chi square test or the Fisher's exact test where appropriate. All the values were expressed as mean $\pm S D$, range, or number (\%). A P value of less than 0.05 was considered statistically significant.

\section{Results}

Over a period of two years, 31 patients (26 males and five females with mean age of $27.48 \pm 7.69$ years and mean weight of $50.87 \pm 5.38 \mathrm{~kg}$ ) with significant hypoxemic changes (SS $\geq 5$; Appendix 2) indicating classical FES were observed preoperatively. Eleven patients had isolated femur fracture another 20 had one or two associated musculoskeletal injuries (NISS > 16). Time intervals between injury and presentation to the institute were shorter than six hours in ten, six to 24 hours in six, 24 to 48 hours in ten, and 48 to 72 hours in five patients. Respiratory discomfort was observed in 23 patients (74.2\%) at the time of admission and in the remaining eight patients (25.8\%) after hospitalization. Petechial hemorrhages were seen in 14 patients (45.2\%). Cerebral symptoms were predominant in five patients while head computed tomographic scan findings were normal in all except one patient in whom it was suggestive of cerebral FES on the second post-operative day. Patent foramen ovale $(5 \mathrm{~mm})$ was detected in one patient. This patient was operated 18 hours after admission and required mechanical ventilatory support with high PEEP for two weeks. At admission, 21 patients (67.8\%) had raised serum lactate levels $(>22 \mathrm{mg} / \mathrm{dL}$ ). The values stabilized back to normal range over next three to five days. Preoperative Murray's LIS was > 2.5 and 0.1 to 2.5 in $5(16.1 \%)$ in 26 patients (83.9\%), respectively. The LIS scores are shown in Figures 1-3.

Out of 16 patients who presented within 24 hours of injury, $13(81.3 \%)$ could be operated during the hypoxemia phase or during the hot phase of FES. Other three patients had fulminant FES manifestation and required hemodynamic support prior to definitive stabilization. Remaining 15 patients who were referred 24 hours after injury with hypoxemic manifestations were operated at the earliest time after ensuring stable respiratory and hemodynamic parameters according to the protocols. Thus, two groups could be defined based upon the timing of surgery after injury, ie, immediate and early surgery group. Patient's demographic characteristics, pattern of injury, perioperative management, and outcome were comparable in these two groups. Except for two patients in early surgery group and three patients in delayed surgery group, all other patients underwent nailing for femur fractures; plating was also performed wherever associated injury was present. Standard techniques of unreamed intramedullary nailing with distal venting and appropriate type of plate fixation was chosen by the operating surgeon. 
Sen RKet al.

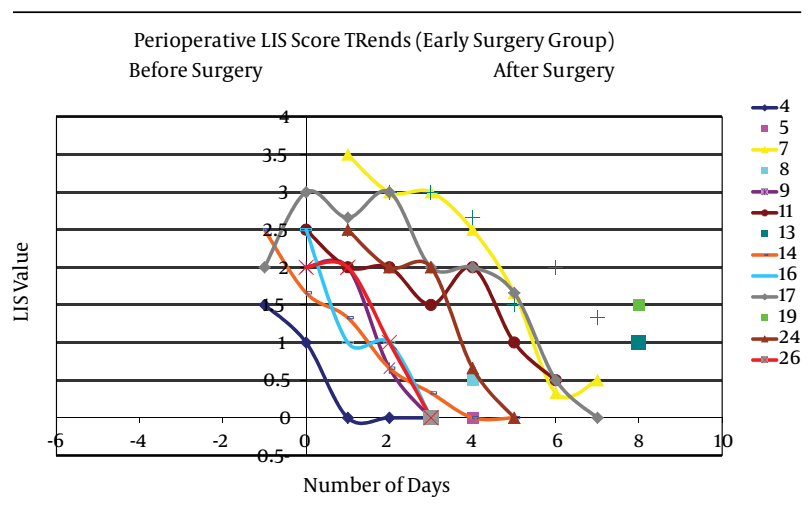

Figure 1. Perioperative Lung Injury Score Trends (Early Surgery Group)

Thus, surgery time varied from 80 to 230 minutes. The LIS improved consistently in all patients in the post-operative period. The total ICU stay was $8.7 \pm 4.8$ days and $4.3 \pm 1.56$ days in XXX and YYY groups, respectively. Post-operative ICU stay had no significant difference between early and delayed surgery groups. Duration of hospitalization was $5.2 \pm 1.9$ days and 17.6 \pm 3.9 days in early and delayed surgery groups, respectively (Table 1 ).

\section{Discussion}

Trauma is a leading cause of morbidity and mortality. In recent years, therapeutic strategies to manage trauma patients have significantly progressed; however, further research to examine different aspects of trauma is still necessary (14). Although the earliest possible surgery is the standard for stabilizing femur fractures $(15,16)$, surgical trauma elicits a stress response of combined endocrine and inflammatory origin $(2,17)$. Thus, surgical interventions needed to fix fractures can increase the patient's embolic burden of marrow fat. This can further influence major organ functions including alveolar gas exchange and the clinical outcome (secondary insult) (7-9). The incidence of clinically manifesting FES reported in literature is $11 \%$ to $29 \%(18,19)$. There is a paucity of data regarding the optimum timing of surgical stabilization in otherwise healthy patients who become significantly hypoxemic.

Various pharmacological and surgical techniques have been developed to block inflammatory cascade of events and to reduce intravasation of fat during surgery. In the present study, physiological parameters such as heart rate, blood pressure, $\mathrm{SO}_{2}$, perioperative GCS, $\mathrm{ABG}, \mathrm{SS}, \mathrm{PaO}_{2} / \mathrm{FiO}_{2}$ ratio, and LIS were continually recorded till the patient were off oxygen therapy. The LIS (9) indicates severity of lung injury. It is aggregate sum of four components, ie, hypoxemia, chest-X-ray, compliance, and PEEP scores. A LIS of zero, 0.1 to 2.5 , and larger than 2.5 stand for no lung injury, moderate lung injury, and severe lung injury, respectively. The $\mathrm{PaO}_{2} / \mathrm{FiO}_{2}$ ratio or oxygenation ratio quantifies extent of lung injury. Values less than $300 \mathrm{mmHg}$ indicate acute lung injury and values less than $200 \mathrm{mmHg}$ with bilateral

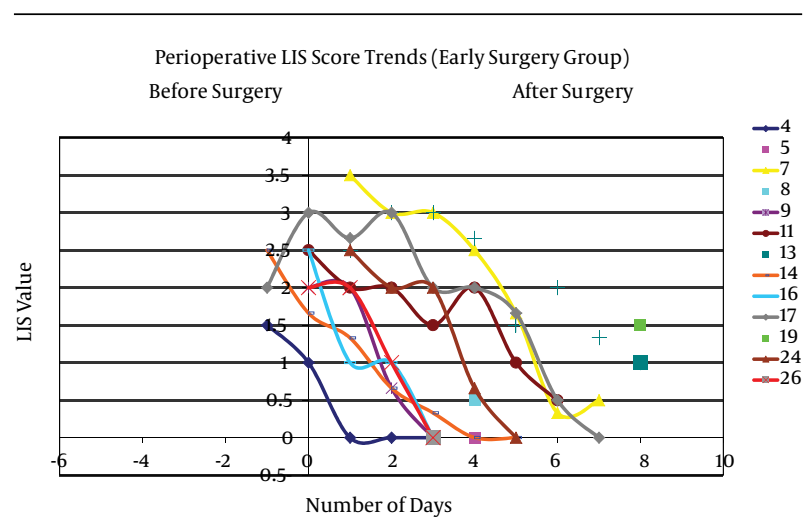

$\overline{\text { Figure 2. Perioperative Lung Injury Score Trends (Delayed Surgery Group) }}$

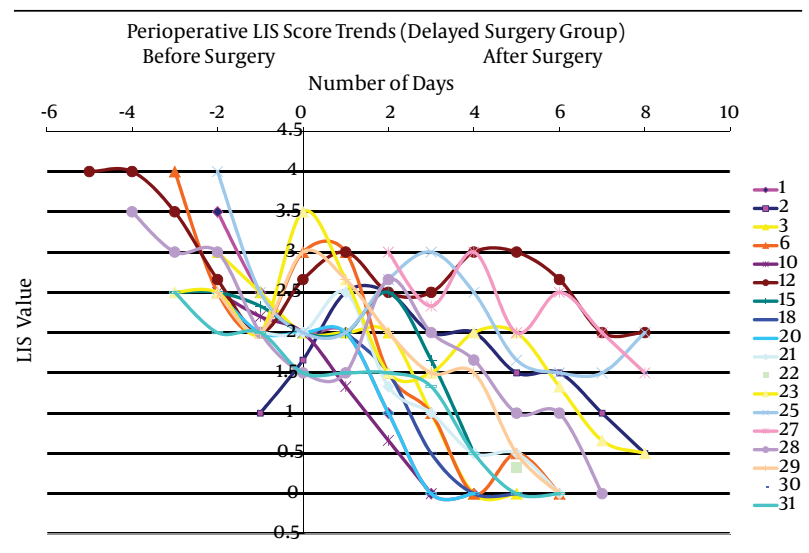

Figure 3. Perioperative Lung Injury Score Trends (All Patients)

chest infiltrates and noncardiogenic pulmonary edema suggest acute respiratory distress syndrome. In the present study, stable hemodynamics without inotropes, serum hemoglobin of more than $10 \mathrm{gm} / \mathrm{dL}$, normal serum electrolytes, coagulation profile, hepatorenal parameters, and $\mathrm{PaO}_{2} / \mathrm{FiO}_{2}$ of larger than $200 \mathrm{mmHg}\left(\mathrm{FiO}_{2}<0.5\right.$, PEEP $<8$ $\mathrm{cm} \mathrm{H}_{2} \mathrm{O}$ ) with LIS values between 0.5 and 2.5 were ensured. Patients with associated cerebral or thoracoabdominal trauma, myocardial insufficiency (myocardial infarction or congestive heart failure), diabetes mellitus, allergy to colloids, hepatorenal disorders, pathological fractures, and pregnancy were not enrolled. The outcome of surgical stabilization was evaluated in patients who were operated after meeting these predefined hemodynamic and respiratory criteria. Satisfactory results were observed using this defined protocol. None of the patients had worsening in their clinical status and there was no readmission/mortality within six weeks and subsequent follow-up after six months.

In conclusion, appropriate surgical stabilization could be safely performed during established post-traumatic hypoxemia when serial assessments had ruled out fulminant pathology and associated injuries. A predefined multidisciplinary criteria-based approach to maintain hemodynamics and respiratory parameters might improve patient satisfaction and final outcome. 
Sen RKet al.

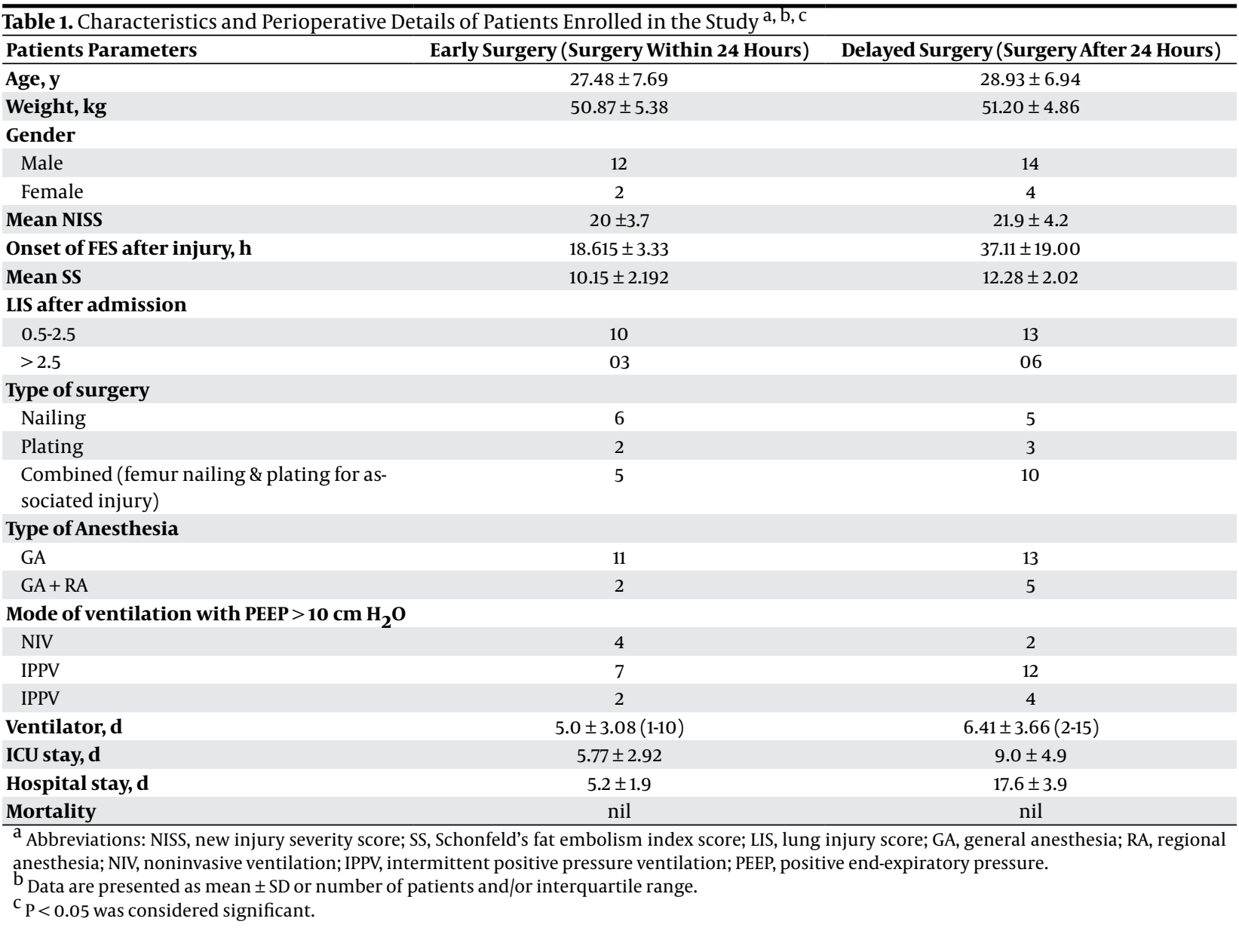

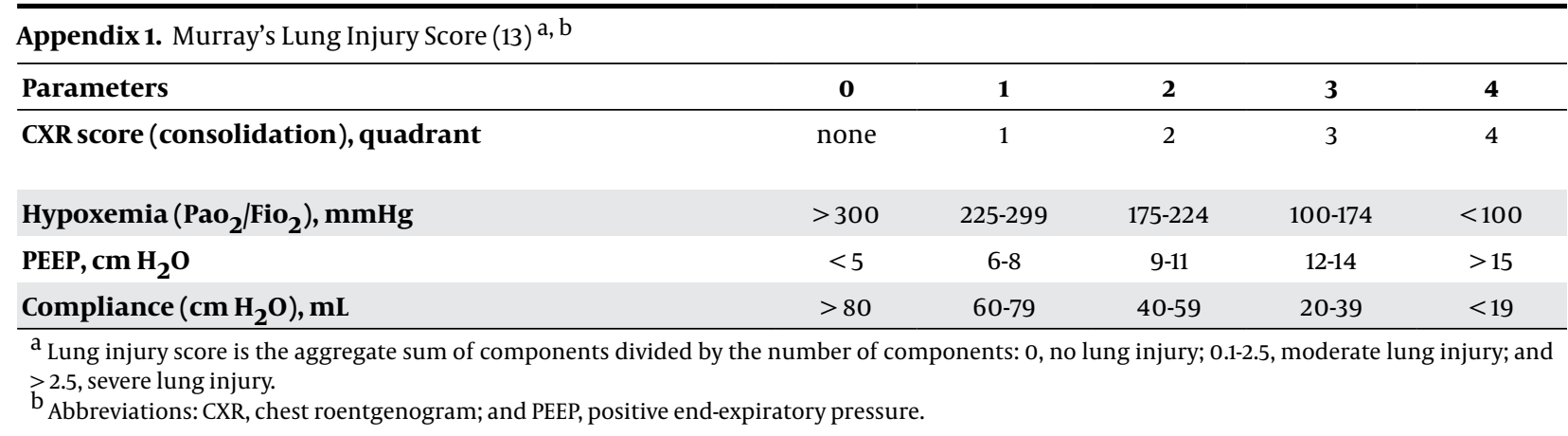

\begin{tabular}{lc}
\hline Appendix 2. Schonfeld's Fat Embolism Index $(12)^{\mathrm{a}}$ \\
\hline Symptoms & Score \\
\hline Petechiae & 5 \\
\hline Diffuse alveolar infiltrates & 4 \\
\hline Hypoxemia $\left(\mathbf{P a O}_{\mathbf{2}}<\mathbf{9 . 3} \mathbf{K p a}\right)$ & 3 \\
Confusion & 1 \\
Fever $>\mathbf{3 8} \mathbf{C}^{\circ} \mathbf{C}$ & 1 \\
Heart rate $>\mathbf{1 2 0} / \mathbf{m i n}$ & 1 \\
\hline Respiratory rate $>\mathbf{3 0} / \mathbf{m i n}$ & 1 \\
\hline
\end{tabular}

${ }^{\mathrm{a}}$ Patients were labeled as hypoxemic if $\mathrm{PaO}_{2}$ in room air was less than $70 \mathrm{mmHg}$ and as clinical fat embolism syndrome if Schonfeld's fat embolism index score was more than five.

\section{Authors' Contributions}

Study concept and design: Prof Ramesh Kumar Sen; collection of data: Prof Puri, Dr Indu Sen, Dr Anil Pratap, and Dr Nirmal Raj; analysis and interpretation of data: Prof Ramesh Sen, Dr Indu Sen, and Dr Anil Pratap; drafting of the manuscript: Dr Indu Sen; critical revision of the manuscript for important intellectual content: Prof Ramesh Kumar Sen, Prof Goverdhan Dutt Puri, and Dr Indu Sen.

\section{Funding/Support}

No financial or material support was received for the research and work conducted in this study by any funding source. 
Sen RKet al.

\section{References}

1. Sinha P, Bunker N, Soni N. Fat embolism-An update. Curr Anaesth Crit Care. 2010;21(5):277-81.

2. Kohl BA, Deutschman CS. The inflammatory response to surgery and trauma. Curr Opin Crit Care. 2006;12(4):325-32.

3. Riska EB, von Bonsdorff H, Hakkinen S, Jaroma H, Kiviluoto O, Paavilainen T. Prevention of fat embolism by early internal fixation of fractures in patients with multiple injuries. Injury. 1976;8(2):110-6.

4. Behrman SW, Fabian TC, Kudsk KA, Taylor JC. Improved outcome with femur fractures: early vs. delayed fixation. J Trauma. 1990;30(7):792-7.

5. Pinney SJ, Keating JF, Meek RN. Fat embolism syndrome in isolated femoral fractures: does timing of nailing influence incidence? Injury. 1998;29(2):131-3.

6. Bone LB, Johnson KD, Weigelt J, Scheinberg R. Early versus delayed stabilization of femoral fractures: a prospective randomized study.1989. Clin Orthop Relat Res. 2004;(422):11-6

7. Moore FA, Moore EE. Evolving concepts in the pathogenesis of postinjury multiple organ failure. Surg Clin North Am. 1995;75(2):257-77.

8. Giannoudis PV, Smith RM, Bellamy MC, Morrison JF, Dickson RA, Guillou PJ. Stimulation of the inflammatory system by reamed and unreamed nailing of femoral fractures. An analysis of the second hit. J Bone Joint Surg Br. 1999;81(2):356-61.

9. Pape HC, Schmidt RE, Rice J, van Griensven M, das Gupta R, Krettek C, et al. Biochemical changes after trauma and skeletal surgery of the lower extremity: quantification of the operative burden. Crit Care Med. 2000;28(10):3441-8.

10. White T, Petrisor BA, Bhandari M. Prevention of fat embolism syndrome. Injury. 2006;37 Suppl 4:S59-67.

11. Osler T, Baker SP, Long W. A modification of the injury severity score that both improves accuracy and simplifies scoring. J Trauma.1997;43(6):922-5.

12. Schonfeld SA, Ploysongsang Y, DiLisio R, Crissman JD, Miller E, Hammerschmidt DE, et al. Fat embolism prophylaxis with corticosteroids. A prospective study in high-risk patients. Ann Intern Med.1983;99(4):438-43.

13. Murray JF, Matthay MA, Luce JM, Flick MR. An expanded definition of the adult respiratory distress syndrome. Am Rev Respir Dis. 1988;138(3):720-3.

14. Fakharian E. Trauma research and its importance. Arch Trauma Res. 2012;1(1):1-2.

15. Pape HC, Tornetta P, 3rd, Tarkin I, Tzioupis C, Sabeson V, Olson SA. Timing of fracture fixation in multitrauma patients: the role of early total care and damage control surgery. J Am Acad Orthop Surg. 2009;17(9):541-9.

16. Kwiatt ME, Seamon MJ. Fat embolism syndrome. Int J Crit Illn Inj Sci. 2013;3(1):64-8.

17. Filomeno LTB, Carelli CR, Silva NCL, Barros Filho TEP, Amatuzzi MM. Fat embolism: a review for current orthopaedics practice. Acta Orthop. 2005;13(4):196-208.

18. Fabian TC, Hoots AV, Stanford DS, Patterson CR, Mangiante EC. Fat embolism syndrome: prospective evaluation in 92 fracture patients. Crit Care Med.1990;18(1):42-6.

19. Taviloglu K, Yanar H. Fat embolism syndrome. Surg Today. 2007;37(1):5-8. 